\section{Vorbild mit Schwächen}

\author{
Ökologische Modernisierung ist nicht nur ein politisches Schlagwort der Nach- \\ wendezeit - ihre Ergebnisse können seit 1989/90 in den neuen Bundeslän- \\ dern beobachtet werden. Dies gilt insbesondere für die alten industriellen Ker- \\ ne der DDR wie Bitterfeld, Buna und Leuna und ihre Privatisierung durch die \\ Treuhandanstalt. Was sind die Stärken und Schwächen der gewählten Moder- \\ nisierungsstrategie, welche Konsequenzen ergeben sich für die Politik? \\ Anhand eines Beispiels aus dem Chemiedreieck lassen sich auch Lehren für \\ Osteuropa ziehen.
}

$\mathrm{E}$

Von Christian Preuschoff

Aandere Standorte steht: ein seit 1936 betriebener petrochemischer Komplex, der in der DDR bis zur Wende 1989/90 volkseigener Betrieb war. Der Betrieb bot über 20.000 Menschen Arbeit, wobei die Produktionsanlagen größtenteils veraltet und die Produkte nicht konkurrenzfähig waren. Luft, Gebäude, Anlagen, Boden und Grundwasser auf dem Gelände waren außergewöhnlich stark verunreinigt.

Nach der Wende 1989/90 ging das Chemiewerk in den Besitz der Treuhandanstalt (THA) über, die als Bundesinstitution die Aufgabe der Privatisierung übernahm. Aufgrund der vermuteten enormen Sanierungskosten von ca. einer Milliarde Mark wurde die Sanierung des Chemiestandortes 1992/93 von staatlicher Seite zum sogenannten ökologischen Großprojekt erklärt. Dies verpflichtete die beiden (staatlichen) Hauptgeldgeber - THA bzw. Bund auf der einen Seite und neue Bundesländer auf der anderen Seite - zur gemeinsamen Finanzierung des Großprojektes, und zwar im Verhältnis $75 \mathrm{zu} 25$.

Im Ergebnis der Privatisierungsbemühungen der nunmehr zur Bundesanstalt für vereinigungsbedingte Sonderaufgaben (BvS) mutierten THA wurde 1995 ein ausländischer Investor aus dem Chemiesektor gefunden. Dieser gewährleistete einen Weiterbetrieb des Werkes nach umfassender technischer und ökologischer Modernisierung und eine Integration in einen internationalen Firmenverbund.

Der Privatisierungsvertrag sah für die Restrukturierung staatliche Subventionen in Milliardenhöhe vor, wobei die bereits im Rahmen des ökologischen Großprojektes vorgesehenen Finanzierungspflichten von BvS und Land ihre Geltung behielten. Dadurch war eine strenge
Kontrolle der Beihilfen vertraglich abgesichert. Die Finanzierungspflicht bei der ökologischen Modernisierung beschränkte sich dabei im wesentlichen auf Gefahrenabwehrmaßnahmen.

Die technische und ökologische Modernisierung begann bereits 1992, intensiv aber erst ab 1995 als Folge des ehrgeizigen Investitionsprogrammes des Erwerbers. Das Projekt erfuhr seine besondere Prägung durch die parallele Abarbeitung der üblicherweise sequenziell ablaufenden Vorgänge Gebäuderïckbau, Altlastensanierung und Neubau. Als ökologische Modernisierungsschwerpunkte kristallisierten sich die Sanierung von einigen hoch quecksilberkontaminierten Anlagen, Gebäuden und Bodenbereichen, die Grundwassererkundung und -sanierung sowie die Sanierung der riesigen Betriebsdeponie heraus. Dabei war auch aus westlicher Sicht verwaltungstechnisch anspruchsvolles Handeln der Landesbehörden gefordert, zum Beispiel bei Freistellungsbescheiden nach dem Umweltrahmengesetz, Abbruchgenehmigungen, Genehmigung von Zwischenlagern, Entsorgungsgenehmigungen, der Festlegung von Wiedereinbauwerten, Sanierungszielen und Gewässerbewirtschaftungszielen sowie öffentlichen und EU-weiten Ausschreibungen.

Bis heute wurden etwa 90 Prozent der vorgesehenen Investitions- und Rückbaumaßnahmen realisiert. Bis zum Jahresende 2000 werden voraussichtlich alle Neubau- und Rückbaumaßnahmen abgeschlossen sein und Subventionen in einstelliger Milliardenhöhe geflossen sein. Von den ökologischen Sanierungsmaßnahmen sind die Erkundungs- und Vorplanungsarbeiten fast vollständig, die Quecksilbersanierung zu 80 Prozent abgeschlossen. Zum Jahresende werden Subventionen von 100 bis 200 Millionen Mark verbraucht sein. Als Sanierungsschwerpunkte verbleiben dann noch die Grundwasser- und die Deponiesanierung. Während die Sanierung und Stillegung der Betriebsdeponie durch den begrenzten Weiterbetrieb selbst erwirtschaftet werden könnte, ist die Grundwassersanierung als schwierigste und langwierigste Aufgabe noch kaum kalkulierbar. Mit Kosten im zwei- bis dreistelligen Millionenbereich, allerdings über viele Jahre verteilt, ist aber zu rechnen. Diese Restaufgaben der ökologischen Modernisierung des Standortes sollen nach dem Willen der BvSFührung auf das entsprechende Bundesland übertragen werden, damit die BvS ihre Aufgabe plangemäß abschließen kann.

Ist das Vorgehen der THA/BvS erfolgreich gewesen, d.h. ist tatsächlich eine nachbaltige ökologische und technische Modernisierung des ostdeutschen Industriestandortes gelungen und damit der gesamten Region eine Wachstumschance eröffnet worden? Eine endgültige Beantwortung dieser Frage dürfte vermutlich erst in einigen Jahren möglich sein, aber eine Betrachtung der Vor- und Nachteile der geschilderten Modernisierungsstrategie sollte eine erste Einschätzung zulassen.

\section{> Stärken der Strategie ...}

1. Es wurden zwar enorme staatliche Beihilfen für die Modernisierung gewährt, aber der Staat bzw. BvS nutzte seine Möglichkeiten zur Kostenkontrolle und Kostenoptimierung. Bereits jetzt zeichnet sich durch diese konsequente Beihilfekontrolle bei der ökologischen Modernisierung eine Kostenersparnis für den Bund und die Länder um 30 bis 40 Prozent und damit um mehrere 100 Millionen Mark ab. Dies rechtfertigt auch den Aufwand für eine Kontrolle.

2. Die Organisation des ökologischen Großprojektes bzw. die Dreiecksbeziehung Bund/BvS Land - Investor zwang alle Parteien zur Kommunikation und zur gemeinsamen Suche nach Kostenoptimierungspotenzialen und tragfähigen Kompromissen. Dadurch wurde trotz teilweise unterschiedlicher Interessenlagen sowohl das gegenseitige Verständnis und Vertrauen gefördert, als auch tatsächlich oft eine kostenoptimale und verhältnismäßige technische Sanierungslösung gefunden.

3. Die Zielstrebigkeit und das Know-how eines privaten Investors wurde zur Modernisierung genutzt und damit die Modernisierung erheblich beschleunigt. 


\section{D... und Schwäichen}

1. Vertraglich war nur eine geringe finanzielle Eigenbeteiligung des Investors vorgesehen und statt dessen die fast vollständige staatliche Subvention, wodurch das Interesse des Investors an einer Kostenoptimierung erheblich gemindert war.

2. Eine Beschränkung der Finanzierung auf Gefahrenabwehrmaßnahmen war zwar kostensparend, aber nicht unbedingt ökologisch nachhaltig. Gerade im Grundwasser- und Gewässerschutz ist eine solche Beschränkung angesichts der Kostbarkeit und Knappheit der Ressource Wasser für künftige Generationen bedenklich und zeugt von eher kurzfristigem Denken.

3. Die Dreiecksbeziehung Bund/BvS - Land Investor verbunden mit dem Einstimmigkeitsprinzip und einer bürokratischen Organisation im ökologischen Großprojekt führte zu einem Konsenszwang, der angesichts der gegensätzlichen Interessen oft zu ungewolltem Projektstillstand oder Verzögerungen führte. Dabei trug die einmalige Machtstellung der THA/BvS, die ja dem Bundesfinanzministerium unterstand und als Bundesverwaltung über das Vermögen der neuen Bundesländer entschied, zu einem erheblichen Übergewicht des Bundeseinflusses gegenüber den neuen Bundesländern bei. Dies störte zwangsläufig das gesamte, auf politischem und finanziellen Gleichgewicht basierende, föderale System und verstärkte bei den neuen Bundesländern das ohnehin vorhandene Gefühl der Bevormundung durch die ,reiche (westliche) Bundesrepublik".

4. Der Faktor Mensch wurde gänzlich vernachlässigt, die unter dem DDR-Regime gewachsenen Erfahrungen und Werte der Ostdeutschen wurden vollkommen negiert. So ist es nicht verwunderlich, dass die fortwährende Kontrolle der Beihilfen durch die BvS und die Gremien des ökologischen Großprojektes von Ostdeutschen in erster Linie als Bevormundung wahrgenommen wurde. Dies entsprach der Bevormundung in der DDR, der sich die meisten entronnen wähnten, und weckte entweder Widerstände oder wurde nach altem Muster als „Vorgabe von oben“ widerspruchslos akzeptiert. In keinem Fall wurde dadurch das für eine nachhaltige ökologische Modernisierung erforderliche Umdenken und eigenverantwortliche Handeln gefördert. Ebensowenig verwundert es, dass die Ostdeutschen nicht über Nacht die Vielzahl von westlichen Rechts- und Verwaltungsvorschriften, die bei einer dermaßen komplizierten ökologischen Sanierung $\mathrm{zu}$ beachten waren, verstanden und danach handelten. Dies hätte eine langjährige Verwaltungserfahrung vorausgesetzt, die die meisten 0stdeutschen in der kurzen Zeit nicht nachholen konnten. Auch die bescheidene Unterstiitzung durch westliche Beamte konnte dieses Defizit nicht ausgleichen, so dass die Verwaltungen einer zügigen Modernisierung oft eher im Wege standen.

\section{Zweifel an der Nachhaltigkeit}

Obwohl die schnelle Erneuerung des Standortes und die deutlichen Kosteneinsparungen durchaus für die gewählte Strategie sprechen, sind angesichts der gravierenden Nachteile Zweifel an der Nachhaltigkeit der Modernisierung und damit Kritik an der Strategie angebracht. Insbesondere die Vernachlässigung des Faktors Mensch ist ein entscheidendes Moment, der einer dauerhaften und nachhaltigen Modernisierung des Standortes, ja der Region, im Wege steht. Solange die Betroffenen den technisch-ökologischen Modernisierungsprozess nicht als Bewusstseins- und Verhaltenswandel vollziehen, kann es nicht zu einem echten Wandel und einer dauerhaften Ökologisierung kommen. Bei diesem mentalen Wandel wurde den Menschen bisher zu wenig Unterstïtzung angeboten. Im Gegenteil, einseitige finanzielle Unterstützung und deren Kontrolle förderten das bereits von der DDR aufgebaute Abhängigkeitsgefühl und hemmten die Entwicklung eines eigenen ökologisch-demokratischen Bewusstseins. Dadurch konnte die eigentliche Stärke der bisherigen Modernisierungsstrategie, nämlich die vertrauensbildende Kommunikation zwischen den unterschiedlichen Parteien, nicht voll motivierend auf alle Beteiligten wirken.

\section{Politische Schlussfolgerungen}

Die ökologische Modernisierungsstrategie offenbart einen starken Einfluss westlicher Denkmuster, die den Modernisierungsprozess wesentlich schwächen. Dies ist die Folge einer wenig durchdachten politischen Vereinigungsstrategie, die bis heute außer einer schnellen Privatisierung keine Strategie zu kennen scheint. Das heißt umgekehrt: Wird eine neue Vereinigungsstrategie entwickelt, können auch die Schwächen der ökologischen Modernisierungen ausgeglichen werden. Dies ist gerade auch im Hinblick auf die Eingliederung der Reformstaaten Osteuropas in die EU von Bedeutung.
Die Entscheidungsträger in Politik und Wirtschaft sollten zunächst den Mut haben, die bisherigen Schwächen als solche zu benennen und die Strategie wo erforderlich zu verändern oder ganz zu verlassen und neue Wege zu gehen.

Weiter sollte eine solche Strategie den Faktor Mensch einbeziehen. Das bedeutet:

- Abkehr von rein finanzieller Beihilfe und deren Kontrolle. Statt dessen Zugeständnis von mehr Eigenverantwortung bei gleichzeitiger ideell-personeller Unterstützung durch den Westen. Mit der Übertragung der ökologischen Restaufgaben auf die neuen Länder wurde bereits ein Schritt zu mehr Eigenverantwortung der neuen Länder gegangen. Doch sollte man hier nicht stehen bleiben, sondern auch personelle Unterstïtzung mit partnerschaftlichem Ansatz bei der Bewältigung der Aufgaben bieten.

- Dies bedeutet nicht, finanzielle Beihilfen für die Modernisierung Ostdeutschlands generell einzustellen. Subventionen für ökologische Sanierungen sind aufgrund der hohen Kosten unumgänglich. Vielmehr sollte eingegrenst werden, welche Projekte gefördert werden und ob weiterhin Großinvestoren und Großprojekte in einem enormen Ausmaß von Beibilfen profitieren sollen. Die neuen technologischen Entwicklungen auf dem Sektor der Informations- und Nanotechnologie lassen eher den Erfolg kleinerer Standorte und Projekte vorausahnen.

- Eine Strategie, die den Faktor Mensch einbezieht, setzt voraus, dass westdeutsche Politik Ostdeutschland als eigenständige Wertewelt mit einer eigenen Identität wahrnimmt und respektiert. Zugleich sollten die Wertunterschiede zur kritischen Reflexion und Diskussion der alten bundesrepublikanischen Prinzipien und Gewohnheiten wie zum Beispiel Föderalismusprinzip/ Bund-Länder-Abhängigkeiten und Fürsorgeprinzip genutzt werden. Erst im Zuge eines solchen Prozesses kann eine gesamtdeutsche Identität entstehen.

\section{Der Autor}

Christian Preuschoff war sieben Jahre als Projektmanager im Direktorat Umweltschutz/Altlasten der THA/BvS tätig und arbeitet jetzt als selbständiger Berater für Veränderungsmanagement. Kontakt: Christian Preuschoff - Management Consultant, Filandastr. 1, 12169 Berlin. Tel. 030/ 797417-57, Fax: -58, E-mail: cpreuschoff@gmx.de 
(c) 20I0 Authors; licensee IÖW and oekom verlag. This is an article distributed under the terms of the Creative Commons Attribution Non-Commercial No Derivates License (http://creativecommons.org/licenses/by-nc-nd/3.o/), which permits unrestricted use, distribution, and reproduction in any medium, provided the original work is properly cited. 